\title{
SOLUTIONS TO LYAPUNOV STABILITY PROBLEMS OF SETS: NONLINEAR SYSTEMS WITH DIFFERENTIABLE MOTIONS
}

\author{
LJUBOMIR T. GRUJIC \\ Department of Electrical Engineering \\ University of Natal \\ Rm. 1-05, Elec. Eng. Bldg. \\ King George V Avenue, Durban 4001, South Africa
}

(Received January 23, 1991 and in revised form April 28, 1993)

\begin{abstract}
Time-invariant nonlinear systems with differentiable motions are considered. The algorithmic necessary and sufficient conditions are established in various forms for one-shot construction of a Lyapunov function, for asymptotic stability of a compact invariant set and for the exact determination of the asymptotic stability domain of the invariant set.

The classical conditions are expressed in terms of existence of a system Lyapunov functions. The conditions of theorems presented herein are expressed via properties of the solution $v$ to $\dot{v}=-p$, or of the solution $w$ to $\dot{w}=-(1-w) p$, for arbitrarily selected $p \in P(S ; f)$ or $p \in P^{1}(S ; f)$, where families $P(S ; f)$ and $P^{1}(S ; f)$ are well defined. The equation $\dot{v}=-p$, or its equivalent $\dot{w}=-(1-w) p$, should be solved only for one selection of the function $p$.
\end{abstract}

Key Words and Phrases: Nonlinear Systems, Lyapunov Functions, and asymptotic stability. 1991 Mathematics Subject Classification Codes: 34A34, 93D05, 93D20.

\section{INTRODUCTION}

The fundamental classical problem of the Lyapunov stability theory [5] has been that of the exact one-shot construction of a system Lyapunov function. This is a consequence of the conditions for asymptotic stability because they are expressed for nonlinear systems via existence of a Lyapunov function. Such classical criteria for asymptotic stability of a set were proved by Zubov [7, p. 204], Bhatia and Szöge [1, p. 207], and La Salle [4, p. 32].

The open problems are the following:

- What are the necessary and sufficient conditions for asymptotic stability of a compact invariant set $J$, which are not expressed via existence of a Lyapunov function?

- What are the necessary and sufficient conditions for one-shot algorithmic construction of a Lyapunov function?

- What are the necessary and sufficient conditions for exact one-shot determination of the asymptotic stability domain of the set $J$ ? The notion of the asymptotic stability domain is defined in the Appendix by following [2], [3].

All three problems are solved in various forms in what follows for a large class of time-invariant nonlinear systems. 


\section{NOTATION}

Capital Roman letters will denote sets and spaces. $J$ will be an invariant set of a system, $J \subset R^{n}$. Its neighborhood will be denoted by $A(J)_{2} N(J)$ or $S(J)$, and its $\delta$-neighborhood will be designated by $B_{0}(J)$, $B_{0}(J)=\{x: \rho(x, J)<\delta\}$, where $\rho(x, J)=\inf \{\|x-y\|: y \in J\}$ with $\|x\|-\left(x^{T} x\right)^{1 / 2}$. Notice that $J \subset A(J)$ and $J \subset B_{0}(J)$. The closure, interior and boundary of a set $J$ are denoted by $\bar{J}, j$ and $\alpha$. The empty set is $\varnothing$.

$D_{s}(J), D_{a}(J)$ and $D(J)$ will be used for the domain of stability of $J$, the domain of attraction of $J$ and the domain of asymptotic stability of $J$, respectively. Their definitions are given in the Appendix by referring to [2], [3].

A motion of the system to be considered is denoted by $\underline{x}\left(t ; x_{0}\right)$ with $\underline{x}\left(0 ; x_{0}\right)=x_{0}$. If $v: R^{n} \rightarrow R$ is differentiable then $d v(x) / d t=\dot{v}(x)$ is the Eulerian derivative of $v$ along system motions.

Other notation will be explained in the sequel.

\section{SYSTEM DESCRIPTION}

A system to be studied is described by

$$
\begin{gathered}
\frac{d x}{d t}=f(x), \\
t \in R, \quad x \in R^{n}, f: R^{n} \rightarrow R^{n} .
\end{gathered}
$$

It is accepted that the system possesses one of the next two smoothness properties.

Weak Smoothness Property:

(i) There is an open neighborhood $S(J)$ of a compact invariant set $J$ of the system (3.1ab) such that for every $x_{0} \in[S(J)-\dot{J}]$ :

(a) the system has the unique solution $\underline{x}\left(t ; x_{0}\right)$, and

(b) the motion $\underline{x}\left(t ; x_{0}\right)$ is defined, continuous and differentiable in $\left(t, x_{0}\right) \in I_{0} x[S(J)-\dot{J}]$, $I_{0}=I_{0}\left(x_{0}\right) \subseteq R_{+}$and $I_{0} \neq \phi$.

(ii) For every $x_{0} \in\left[R^{n}-S(J)\right]$ every motion $\underline{x}\left(t ; x_{0}\right)$ of the system (3.1ab) is continuous in $t \in I_{0}$.

Strong Smoothness Property:

(i) The system (3.1ab) possesses the Weak Smoothness Property.

(ii) If the boundary $2 S(J)$ of $S(J)$ is non-empty then every motion of the system (3.1ab) passing through $x_{0} \in \partial S(J)$ obeys $\inf \left\{\rho\left[\underline{x}\left(t ; x_{0}\right), S(J)\right]: t \in R_{+}\right\}>0$ for every $x_{0} \in \partial S(J)$.

\section{LYAPUNOV FUNCTION GENERATION AND DETERMINATION OF THE ASYMPTOTIC STABILITY DOMAIN FOR THE SYSTEM WITH STRONG SMOOTHNESS PROPERTY}

A function $v: R^{n} \rightarrow R$ will be called positive definite with respect to $J$ if and only if there is a neighborhood $A(J)$ of $J$ such that

(i) $v(x)$ is continuous in $x \in A(J)$,

(ii) $v(x)>0$ for every $x \in[A(J)-\bar{J}]$,

(iii) $v(x)=0$ for every $x \in \bar{J}$.

A function $v$ is positive definite on $N(J)$ with respect to $J$ if and only if the preceding conditions (i)-(iii) hold for $A(J)=N(J)$. 
We shall write $A, B_{(\cdot)}, D_{(\cdot)}, N$ and $S$ instead of $A(J), B_{(\cdot)}(J), D_{(\cdot)}(J), N(J)$ and $S(J)$, respectively, as soon as $J$ is known and fixed. In the sequel the set $J$ is assumed known and fixed.

In order to generate a $v$-function (Lyapunov function) for the system (3.1ab) the following definition is introduced.

Definition 1. (i) $P(S ; f)$ is the family of all functions $p: R^{n} \rightarrow R$ obeying 1$\left.)-3\right)$,

1) $\quad p$ is differentiable on $S$ and positive definite on $S$ with respect to $J$,

2) for any $\alpha>0$ such that $\bar{B}_{\alpha} \subset S$ there is $\beta>0, \beta=\beta(\alpha ; p ; S)$, satisfying $\inf \left[p(x): x \in\left(S-B_{a}\right)\right]-\beta$,

3) there is $\mu \in] 0,+\infty[, \mu=\mu(p ; f)$, such that there exists a solution $v$ to the following system determined along motions of the system (3.1ab),

$$
\begin{gathered}
\frac{d}{d t} v(x)=[\operatorname{grad} v(x)]^{T} f(x)=-p(x) \\
v(x)=0, \quad \forall x \in J,
\end{gathered}
$$

which is defined and continuous in $x \in \bar{B}_{\mu}$.

(ii) $P^{1}(S ; f)$ is the family of all functions $p \in P(S ; f)$ for which the solution function $v$ to (4.1ab) is also differentiable on $\bar{B}_{\mu}$.

Notice that $p \in P(S ; f)$ does not imply by itself that the solution function $v$ to $(4.1 a b)$ is positive definite with respect to $J$. In fact, $p \in P(S ; f)$ guarantees only existence of a continuous solution $v$ to (4.12ab) on any small neighborhood $\bar{B}_{\mu}$ of $J$. Therefore, a selection of $p$ to obey $p \in P(S ; f)$ is rather a pure problem of solving (4.1 $a b)$ than a stability problem. Methods for solving $(4.1 a b)$ will not be considered herein.

The condition 2) of Definition 1 means that $p(x)$ does not converge to zero as $x \rightarrow \partial S$ or $\|x\| \Rightarrow+\infty, x \in S$. For example, $p(x)=0$ if $x \in J$ and $p(x)-\left(\|x\|^{2}-1\right)\left(2+\|x\|^{2}\right)$ if $x \notin J$, and $S=B_{1}=\{x: \rho(x, J)<1\}$ with $J=\{x:\|x\|<1\}$ obey the condition 2). But, $p(x)=0$ if $x \in J$ and $p(x)=\left(\|x\|^{2}-1\right)\left(2-\|x\|^{2}\right)$ if $x \notin J$, and $S=B_{1}$ do not fulfill the condition 2). Notice that $p$ is positive definite on $B_{1}$ with respect to $J$ and differentiable on $B_{1}$ in both cases.

Theorem 1. In order for a compact invariant set $J$ of the system (3.1ab) with the Strong Smoothness Property to have the domain $D$ of asymptotic stability and for a set $N, N \subseteq R^{n}$, to be the domain $D: N-D$, it is both necessary and sufficient that

1) the set $N$ is an open connected neighborhood of $J$ and $N \subseteq S$, and

2) (a) for arbitrarily selected function $p \in P(S ; f)$, the equations (4.1ab) have the unique solution $v$ on $N$ with the following properties:

(i) $v$ is positive definite on $N$ with respect to $J$,

(ii) if the boundary $\partial N$ of $N$ is non-empty then $v(x) \rightarrow+\infty$ as $x \rightarrow \partial N, x \in N$, or,

(b) for arbitrarily selected $p \in P^{1}(S ; f)$ the equations (4.1ab) have the unique solution $v$ on $N$ with the following properties:

(i) $v$ is differentiable on $N$ and positive definite on $N$ with respect to $J$, and

(ii) if the boundary $\partial N$ of $N$ is non-empty then $v(x) \rightarrow+\infty$ as $x \rightarrow \partial N, x \in N$.

Proof. Necessity. Let the compact invariant set $J$ of the system (3.1ab) with the Strong Smoothness Property have the asymptotic stability domain $D$. Hence, it also has $D_{a}$ (Definitions A-2 and A-3 of the Appendix), which is a connected open neighborhood of $J$. Evidently, $D_{a} \cap S \neq \phi$. Let $D_{a} \subseteq S$ be first proved. If $2 S=\phi$ then $S=R^{n}$ and $D_{a} \subseteq S$ due to $D_{a} \subseteq R^{n}$. If $\partial S \neq \phi$ then $x_{0} \in \partial S$ and $x_{0}^{*} \in\left(R^{n}-\bar{S}\right)$ will be analyzed separately. In case $x_{0} \in \partial S$ then $x_{0} \notin D_{a}$ due to the Strong Smoothness Property. Hence, $\partial S \cap D_{a}=\phi$ and $\partial S \cap D=\phi$ 
due to $D-D_{a}$ implied by the Weak Smoothness Property (Lemma A-1, Appendix). In case $x_{0}^{*} \in\left(R^{n}-\bar{S}\right)$ then $\underline{x}\left(t ; x_{0}^{*}\right)$ does not converge to $J$ due to the Strong Smoothness Property. Therefore, $x_{0}^{*} \notin D_{a}$ and $D_{a} \cap\left(R^{n}-\bar{S}\right)=\phi$ so that $D \cap\left(R^{n}-\bar{S}\right)=\phi$. From $D \cap S \neq \phi, D \cap \partial S-\phi$ and $D \cap \partial\left(R^{n}-\bar{S}\right)-\phi$ it results that $D \subseteq S$. Let $N=D$. Hence, $N \subseteq S$ and $N$ is open connected neighborhood of $J$, which proves necessity of the condition 1).

$N=D$ implies (Definitions A-1 to A-3) that $I_{0}\left(x_{0}\right)=R_{+}, \forall x_{0} \in N$. Let $p \in P(S ; f),\left[p \in P^{1}(S ; f)\right]$, be arbitrarily selected. Hence, there is a solution $v$ to $(3.1 a b)$, which is defined and continuous on $\bar{B}_{\mu}$, [and differentiable on $\bar{B}_{\mu}$ ], respectively,

$$
v(x) \in C\left(\bar{B}_{\mu}\right), \quad\left[v(x) \in C^{(1)}\left(\bar{B}_{\mu}\right)\right]
$$

Let $\tau \in] 0,+\infty\left[, \tau=\tau\left(x_{0} ; f ; \mu ; J\right)\right.$, be such that for any $x_{0} \in N$ the condition (4.3) holds,

$$
\underline{x}\left(t ; x_{0}\right) \in \bar{B}_{\mu}, \quad \forall t \in[\tau,+\infty] .
$$

Existence of such $\tau$ is implied by $N=D=D_{a}$ (Definition A-2). Besides,

$$
\underline{x}\left(+\infty ; x_{0}\right) \in \bar{J}, \quad \forall x_{0} \in N .
$$

After integrating (4.1.a) and using (4.1.b) together with (4.4) we derive (4.5),

$$
v\left[\underline{x}\left(t ; x_{0}\right)\right]=\int_{1}^{+\infty} p\left[\underline{x}\left(\sigma ; x_{0}\right)\right] d \sigma, \quad \forall\left(t, x_{0}\right) \in R_{+} x N .
$$

Invariance of $D_{a}, D=D_{a}, N=D$, continuity of motions $\underline{x}$ (the weak Smoothness Property), continuity of $p$ on $N$, the definition of $\tau(4.3)$ and compactness of $[t, \tau]$ for any $t \in R_{+}$prove

$$
\left|\int_{t}^{\tau} p\left[\underline{x}\left(\sigma ; x_{0}\right)\right] d \sigma\right|<+\infty, \quad \forall\left(t, x_{0}\right) \in R_{+} x N .
$$

(4.2), (4.3) and $\underline{x}\left(\sigma ; x_{0}\right)=\underline{x}\left[\sigma ; \tau ; \underline{x}\left(\tau ; x_{0}\right)\right] \in \bar{B}_{\mu}$ for $\sigma \in[\tau,+\infty]$ and the condition 3) of Definition 1 yield

$$
\left|\int_{\tau}^{+\infty} p\left[\underline{x}\left(\sigma ; x_{0}\right)\right] d \sigma\right|<+\infty, \quad \forall x_{0} \in N .
$$

Now, (4.5)-(4.7) gives

$$
\left|v\left[\underline{x}\left(t ; x_{0}\right)\right]\right|<+\infty, \quad \forall\left(t, x_{0}\right) \in R_{+} x N,
$$

or, for $t=0$ and $x=x_{0}$,

$$
|v(x)|<+\infty, \quad \forall x \in N .
$$

Differentiability of $\underline{x}$ in $x_{0} \in N, p \in P(S ; f),\left[p \in P^{1}(S ; f)\right]$, invariance of $N=D=D_{a},(4.5)$ and (4.8) prove continuity of $v$ on $N$, [differentiability of $v$ on $N$ ],

$$
v(x) \in C(N), \quad\left[v(x) \in C^{(1)}(N)\right],
$$

respectively. Invariance of $N$, positive definiteness of $p$ on $S$ with respect to $J, N \subseteq S$, and (4.5) imply

$$
v(x)>0, \quad \forall x \in(N-J) .
$$

Now, (4.1a), (4.10) and (4.11) verify positive definiteness of $v$ on $N$ with respect to $J$ [and its differentiability on $N$ ], respectively. Positive definiteness of $p$, uniqueness of the motions $\underline{x}\left(t ; x_{0}\right)$ for every $x_{0} \in S, N \subseteq S$, invariance of $N$ and (4.5) prove uniqueness of the solution $v$ to (4.1 $a b$ ). This completes the proof of necessity of the conditions 2-a-i) and 2-b-i), respectively.

Let $x_{k}$ be a sequence, $x_{k} \rightarrow \hat{x}$ as $k \rightarrow+\infty, \hat{x} \in \partial N, \partial N \propto \phi$, and $x_{k} \in N$. Let $\left.\zeta \in\right] 0,+\infty[$ be arbitrarily selected so that $\bar{B}_{\zeta} \subset N$. Let $T_{k}, T_{k}=T_{k}\left(x_{k} ; \zeta\right) \in[0,+\infty[$, be the first moment satisfying (4.12), 


$$
\underline{x}\left(t ; x_{k}\right) \in \bar{B}_{\zeta}, \quad \forall t \in\left[T_{k},+\infty[.\right.
$$

Such $T_{k}$ exists due to $x_{k} \in N$ and $N=D=D_{a}$ (Definition A-2). Continuity of $\underline{x}\left(t ; x_{0}\right)$ in $\left(t, x_{0}\right) \in R, x N$, $N=D, D=\dot{D}_{a}=D_{a}$ and $D \subseteq S$ imply $T_{k} \rightarrow+\infty$ as $k \rightarrow+\infty$. Let $m$ be such a natural number that $x_{k} \in\left(N-\bar{B}_{\zeta}\right)$ for all $k=m, m+1, \ldots$. Such $m$ exists because $N$ is open, $\bar{B}_{\zeta} \subset N$ and $x_{k} \rightarrow \partial N$ as $k \rightarrow+\infty$. Let $\alpha$ be introduced by

$$
\alpha=\min \left[p(x): x \in\left(N-\bar{B}_{\zeta}\right)\right] .
$$

$p \in P(S ; f),\left[p \in P^{1}(S ; f)\right]$, guarantees (due to the condition 2) of Definition 1)

$$
\alpha \in] 0,+\infty[\text {. }
$$

From (4.6) and (4.13) we derive (4.15) by setting $t=0$ and $\underline{x}\left(0 ; x_{k}\right)=x_{k}$,

$$
v\left(x_{k}\right) \geq \int_{0}^{k_{k}} \alpha d \sigma+\int_{T_{k}}^{+\infty} p\left[\underline{x}\left(\sigma ; x_{k}\right)\right] d \sigma, \quad x_{k} \in(N-J), \quad k=m, m+1, \ldots
$$

Invariance of $N=D=D_{a}$, positive definiteness of $p$ on $S$ with respect to $J$ (the condition 1) of Definition 1), $N \subseteq S$ and (4.16) imply

$$
v\left(x_{k}\right) \geq \alpha T_{k}, \quad x_{k} \in(N-J), \quad k=m, m+1, \ldots
$$

This result, $T_{k} \rightarrow+\infty$ as $k \rightarrow+\infty$ and (4.14) yield $v\left(x_{k}\right) \rightarrow+\infty$ as $x_{k} \rightarrow \partial N, x_{k} \in N$, which proves necessity of the conditions 2-a-ii) and 2-b-ii), respectively.

Sufficiency. Let all conditions of Theorem 1 be valid. Then, the set $J$ is asymptotically stable [1, p. 208], [7, p. 204]. The system (3.1ab) has the domain $D$ of asymptotic stability of $J$ (Definitions A-1 to A-3). The condition 1) implies $N \subseteq S$. Two possible cases will be considered: a) the boundary $\partial N$ of $N$ is empty, and b) $\partial N$ is non-empty.

a) Let $\partial N=\phi$. Then $N=R^{n}$ that obviously implies $N=D=R^{n}$ due to the conditions 2-a-i and 2-b-i).

b) Let $\partial N \neq \phi$. If $\partial S=\phi$ then $S=R^{n}$ so that $D \subseteq S$. If $\partial S \neq \phi$ then $\partial S \cap D=\phi$ due to (ii) of the Strong Smoothness Property. This fact as well as that both $D$ and $S$ are neighborhoods of $J$ prove $D \subseteq S$. In both cases $D \subseteq S$. Let now $\partial D \approx \phi$ and $\partial D=\phi$ be treated separately. If $\partial D \approx \phi$ then the definition of $v$ as the solution to (4.1ab), $D \subseteq S$ and the proof of the necessity part show that $v$ is continuous on $D$ and $v(x) \rightarrow+\infty$ as $x \rightarrow \partial D, x \in D$. These facts, continuity of $v$ on $N, N \subseteq S$, the fact that $D$ and $N$ are connected neighborhoods of $J$ and $v(x) \rightarrow+\infty$ as $x \rightarrow \partial N, x \in N$, imply

$$
N=D
$$

Let now $\partial D=\phi$. Hence, $D=R^{n}$. The solution $v$ to $(4.1 a b)$ is continuous on $D=R^{n}$ as shown in the necessity part. Hence, $|v(x)|<+\infty$ for every $x \in R^{n}$. Since $v(x) \rightarrow+\infty$ as $x \rightarrow \partial N, x \in N$, then $\partial N \cap R^{n}-\phi$ and $N=R^{n}$. Finally, (4.17) holds also in case $\partial D=\phi$, which proves (4.17) in all cases and completes the proof.

From the computational point of view the form of the condition " $v(x) \rightarrow+\infty$ as $x \rightarrow \partial N, x \in N^{n}$ is not suitable. It can be set in another form by utilizing $w$ as used by Vanelli and Vidyasagar [6],

$$
w(x)=1-\exp [-v(x)] \text {. }
$$

Evidently, the following are true:

a) $\quad w$ is defined and continuous [and differentiable] on $S$ if and only if $v$ is defined and continuous [and differentiable], respectively, on $S$,

b) positive definiteness of $v$ on $S$ with respect to $J$ implies positive definiteness of $w$ on $S$ with respect to $J$, and vice versa, 
c) $v \rightarrow+\infty$ implies $w \rightarrow+1$ and vice versa,

d) the equations (4.1 $a b$ ) are equivalent to the following system

$$
\begin{gathered}
\frac{d}{d t} w(x)=-[1-w(x)] p(x), \\
w(x)=0, \quad \forall x \in J .
\end{gathered}
$$

The facts listed above under a) to d) and Theorem 1 directly yield the next result:

Theorem 2. Let the function $v$ be replaced by $w$ and the equations (4.1ab) by the equations (4.19ab) in Definition 1.

In order for a compact invariant set $J$ of the system (3.1ab) with the Strong Smoothness Property to have the domain $D$ of asymptotic stability and for a set $N, N \subseteq R^{n}$, to be the domain $D: N=D$, it is both necessary and sufficient that

1) the set $N$ is an open connected neighborhood of $J$ and $N \subseteq S$, and

2) (a) for arbitrarily selected function $p \in P(S ; f)$, the equations (4.19ab) have the unique solution w on $N$ with the following properties:

(i) $w$ is positive definite on $N$ with respect to J, and

(ii) if the boundary $\partial N$ of $N$ is non-empty then $w(x) \rightarrow+1$ as $x \rightarrow \partial N, x \in N$,

or,

(b) for arbitrarily selected $p \in P^{1}(S ; f)$ the equations (4.19ab) have the unique solution $w$ on $N$ with the following properties:

(i) $\quad w$ is differentiable on $N$ and positive definite on $N$ with respect to $J$, and

(ii) if the boundary $\partial N$ of $N$ is non-empty then $w(x) \rightarrow+1$ as $x \rightarrow \partial N, x \in N$.

\section{GENERATION OF A LYAPUNOV FUNCTION AND DETERMINATION \\ OF THE ASYMPTOTIC STABILITY DOMAIN FOR THE SYSTEMS WITH THE WEAK SMOOTHNESS PROPERTY}

The class of the systems described by (3.1ab) with the Weak Smoothness Property is larger than that with the Strong Smoothness Property. It is not surprising that the conditions of the preceding theorems slightly change for the systems with the Weak Smoothness property as follows.

Theorem 3. In order for a compact invariant set J of the system (3.1 ab) with the Weak Smoothness Property to have the domain $D$ of asymptotic stability and that a subset $N$ of $S$ equals $D: N=D$, it is both necessary and sufficient that

1) the set $N$ is open connected neighborhood of $J$,

2) (a) for arbitrarily selected function $p \in P\left(R^{n} ; f\right)$, the equations (4.1ab) have the unique solution function $v$ on $N$ with the following properties:

(i) $v$ is positive definite on $N$ with respect to $J$,

and

(ii) if the boundary $\partial N$ of $N$ is non-empty then $v(x) \rightarrow+\infty$ as $x \rightarrow \partial N, x \in N$, or,

(b) for arbitrarily selected function $p \in P^{1}\left(R^{n} ; f\right)$, the equations (4.1ab) have the unique solution function $v$ on $N$ with the following properties: 
(i) $v$ is differentiable on $N$ and positive definite on $N$ with respect to $J$, and

(ii) if the boundary $\partial N$ of $N$ is non-empty then $v(x) \rightarrow+\infty$ as $x \rightarrow \partial N, x \in N$.

Proof. Necessity. Let the system (3.1ab) possess the Weak Smoothness Property. Let the system (3.1ab) have the asymptotic stability domain $D$ and let $N=D$, for $N \subseteq S$. Let $p \in P\left(R^{n} ; f\right),\left[p \in P^{1}\left(R^{n} ; f\right)\right]$, be arbitrarily selected. From this point on we should simply repeat the corresponding part of the proof of necessity of the conditions of Theorem 1 in order to show necessity of all conditions of Theorem 3 .

Sufficiency. Let the system (3.1ab) have the Weak Smoothness Property. Let the conditions of Theorem 3 hold. Then, the invariant set $J$ is asymptotically stable. The system $(3.1 a b)$ has the domain $D$ of asymptotic stability of $J$ (Definition A-3), which equals $D_{a}$ (Lemma A-1). Let $x_{0} \in\left(R^{n}-N\right)$. Continuity of $\underline{x}\left(t ; x_{0}\right)$ in $t \in I_{0}$ due to the Weak Smoothness Property, positive definiteness of $p$ on $R^{n}$ due to $p \in P\left(R^{n} ; f\right)$, $\left[p \in P^{1}\left(R^{n} ; f\right)\right]$, negativeness of $\dot{v}(x)$ on $\left(R^{n}-N\right)$ guaranteed by positive definiteness of $p$ on $R^{n}$ and (4.1ab), and the condition 2-a-ii), [2-b-ii)], respectively, imply $\underline{x}\left(t ; x_{0}\right) \in\left(R^{n}-N\right)$ for all $t \in I_{0}$. Hence, $D \subseteq N$. Furthermore, (4.1a) and positive definiteness of $p$ on $R^{n}$ imply (see the proof of the necessity part of Theorem 1) $v(x) \rightarrow+\infty$ as $x \rightarrow \partial D, x \in D$, which together with the condition 2-a-i), [2-b-i)], respectively, implies $\partial D \cap N \neq \phi$. This result, $D \subseteq N$ and the fact that $D$ is a neighborhood of $J$ imply $D-N$ and complete the proof.

The counterpart to Theorem 2 in this framework is the next result that follows directly from Theorem 3 and (4.18).

Theorem 4. Let the function $v$ be replaced by $w$ and the equations (4.1ab) by the equations (4.19ab) in Definition 1.

In order for a compact invariant set $J$ of the system (3.1ab) with the Weak Smoothness Property to have the domain $D$ of asymptotic stability and that a subset $N$ of $S$ equals $D: N-D$, it is both necessary and sufficient that

1) the set $N$ is open connected neighborhood of J, and

2) (a) for arbitrarily selected function $p \in P\left(R^{n} ; f\right)$, the equations (4.19ab) have the unique solution $w$ on $N$ with the following properties:

(i) $w$ is positive definite on $N$ with respect to $J$, and

(ii) if the boundary $\partial N$ of $N$ is non-empty then $w(x) \rightarrow+1$ as $x \in \partial N, x \in N$,

or

(b) for arbitrarily selected function $p \in P^{1}\left(R^{n} ; f\right)$, the equations $(4.19, a b)$ have the unique solution $w$ on $N$ with the following properties:

(i) $w$ is differentiable on $N$ and positive definite on $N$ with respect to $J$, and

(ii) if the boundary $\partial N$ of $N$ is non-empty then $w(x) \rightarrow+1$ as $x \rightarrow \partial N, x \in N$.

\section{GENERATION OF A LYAPUNOV FUNCTION AND ASYMPTOTIC STABILITY}

The classical problem of the Lyapunov stability theory has been the problem of the necessary and sufficient conditions for asymptotic stability (without determination of the asymptotic stability domain). It generated the problem of the necessary and sufficient conditions for an exact, direct and one-shot construction of a system Lyapunov function. The solution to these problems results directly from the proof of Theorem 1 and Theorem 3 in the following form.

Theorem 5. In order for a compact invariant set $J$ of the system (3.1 ab) with the Weak Smoothness Property to be asymptotically stable it is both necessary and sufficient that

1) for arbitrarily selected function p obeying the conditions 1) and 3) of (i) of Definition 1, the equations (4.1ab) have the unique positive definite solution function $v$ with respect to $J$, 
or,

2) for arbitrarily selected function pobeying the conditions 1) and 3) of (i) and (ii) of Definition 1, the equations (4.1ab) have the unique differentiable positive definite solution function $v$ with respect to $J$.

This theorem, Theorem 1 and Theorem 3 show that the condition 2) of (i) of Definition 1 is needed only for the exact determination of the asymptotic stability domain $D$ of $J$.

By making use of (4.18) the solution can be stated in terms of the solution $w$ to (4.19ab).

Theorem 6. Let the function $v$ be replaced by $w$ and the equations (4.1ab) by the equations $(4.19 a b)$ in Definition 1.

In order for a compact invariant set J of the system (3.1ab) with the Weak Smoothness Property to be asymptotically stable it is both necessary and sufficient that

1) for arbitrarily selected function pobeying the conditions 1) and 3) of (i) of Definition 1, the equations (4.19ab) have the unique positive definite solution $w$ with respect to J, or,

2) for arbitrarily selected function pobeying the conditions 1) and 3) of (i) and (ii) of Definition 1, the equations (4.19ab) have the unique differentiable positive definite solution function $w$ with respect to J.

\section{EXAMPLES}

Example 1. Let a simple second order nonlinear system (3.1ab) have the following specific form:

$$
\frac{d x}{d t}=\left(1-\|x\|^{2}\right)\left(100-\|x\|^{2}\right)^{2} x .
$$

The system has the set $S_{e}$ of the equilibrium states,

$$
S_{e}=\{x:\|x\|=0 \text { or }\|x\|=1 \text { or }\|x\|=10\} .
$$

The set $J$,

$$
J=\{x:\|x\| \leq 1\},
$$

is a compact invariant set of the system. From (7.1) and (7.2) it follows that the system possesses the Strong Smoothness Property with the set $S$ given by

$$
S=\{x:\|x\|<10\} .
$$

Let the function $p$ be selected in the form

$$
p(x)=\left\{\begin{array}{cl}
0, & \|x\| \leq 1, \\
\left(\|x\|^{2}-1\right)\|x\|^{2}, & \|x\| \geq 1 .
\end{array}\right.
$$

It is differentiable on $R^{2}$ and positive definite on $R^{2}$ with respect to the set $J$ (7.3). The solution function $v$ to $(4.1 a b)$ for $p$ defined by $(7.5)$ is obtained in the form

$$
v(x)=\left\{\begin{array}{cl}
0, & \|x\| \leq 1, \\
\frac{\|x\|^{2}-1}{198\left(100-\|x\|^{2}\right)}, & \|x\| \geq 1 .
\end{array}\right.
$$

The function $v$ is defined, continuous and differentiable on the set $S(7.4)$. Hence, $p \in P^{1}(S ; f)$. Besides, the function $v$ is positive definite on $S$ with respect to $J(7.3)$ and $v(x) \rightarrow+\infty$ as $x \rightarrow \partial S, x \in S$, where

$$
\partial S=\{x:\|x\|=10\} .
$$

Since the set $S$ is open connected neighborhood of the set $J$ (7.3) then all the conditions of Theorem 1 are satisfied for the set $N=S$ (7.4). This means that the domain $D$ of asymptotic stability of the compact invariant set $J(7.3)$ of the system (7.1) equals $S$, 


$$
D=S=\{x:\|x\|<10\} .
$$

Since the system is simple this result can be easily verified.

Example 2. Let a third order nonlinear system (3.1ab) be described by

$$
\begin{aligned}
\frac{d x}{d t} & =-\frac{2 \cdot 10^{4}\left\{1-\sin \left[\pi\left(2 \cdot 10^{4}\right)^{-1}\left(x^{T} H x-10\right)^{2}\right]\right\}\left(x^{T} H x-10\right)}{\pi \cos \left[\pi\left(2 \cdot 10^{4}\right)^{-1}\left(x^{T} H x-10\right)^{2}\right]} x-f(x), \\
H & =\left[\begin{array}{ccc}
10 & -4 & -6 \\
-4 & 10 & 2 \\
-6 & 2 & 5
\end{array}\right]=H^{T} .
\end{aligned}
$$

The matrix $H$ is positive definite. The closed invariant set $J$ of the system is

$$
J=\left\{x: x^{T} H x \leq 10\right\} .
$$

The system possesses the Strong Smoothness Property with the set $S$ given by

$$
S=\left\{x: x^{T} H x<110\right\} .
$$

The set $S$ (7.9) is open connected neighborhood of $J(7.8)$. Let the function $p$ be accepted in the next form:

$$
p(x)=\left\{\begin{array}{cl}
0 & x \in J \\
4\left(x^{T} H x-10\right)^{2} x^{T} H x, & x \in\left(R^{3}-j\right) .
\end{array}\right.
$$

The function $p$ is defined, continuous and differentiable on $R^{3}$, and positive definite on $R^{3}$ with respect to $J$. It leads to the following solution function $v$ to the equations (4.1ab),

$$
v(x)=\left\{\begin{array}{cl}
0 & , \quad x \in J \\
-\ln \left\{1-\sin \left[\pi\left(2 \cdot 10^{4}\right)^{-1}\left(x^{T} H x-10\right)^{2}\right]\right\}, & x \in\left(R^{3}-J\right) .
\end{array}\right.
$$

The function $v(7.11)$ is defined, continuous and differentiable on $S$. Hence, the function $p$ belongs to $P^{1}(S ; f)$. Furthermore, the function $v(7.11)$ is also positive definite on $S$ with respect to $J$. Besides,

$$
v(x) \rightarrow+\infty \text { as } x \rightarrow \partial S=\left\{x: x^{T} H x=100\right\} .
$$

All conditions of Theorem 1 have been verified. Hence, the system (7.7) has the domain $D$ of asymptotic stability of $J$, which equals $N=S$,

$$
D=S=\left\{x: x^{T} H x<110\right\}
$$

\section{CONCLUSION}

Nonlinear time-invariant systems characterized by the smoothness properties are considered. The problems of the necessary and sufficient conditions for an exact direct construction of a system Lyapunov function, for asymptotic stability of a compact invariant set $J$ and for the exact determination of its asymptotic stability domain are solved algorithmically. This means that the invariant set $J$ is asymptotically stable if and only if the solution $v$ to $\dot{v}=-p(4.1 a)$ with $v(x)=0$ on $J(4.1 b)$ is positive definite [and differentiable] for any $p \in P(S ; f),\left[p \in P^{1}(S ; f)\right]$, respectively. The equation $(4.1 a)$ is to be solved only for one such arbitrarily selected $p$. If the solution $v$ is positive definite with respect to $J$ then $J$ is asymptotically stable. However, if the solution $v$ is not positive definite with respect to $J$ then $J$ is not asymptotically stable. In the latter case there is not sense to try solving $\dot{v}=-p$ with any other function $p$. These statements result from Theorem 5 that together with Theorem 6 opens new direction onto the asymptotic stability analysis.

The complete sets of conditions for the exact determination of the asymptotic stability domain of $J$ are given in various forms of Theorem 1 to Theorem 4 . They establish essentially new approach to solving the Lyapunov stability problems. 
If the set $J=\{0\}$ and the system (3.1ab) is linear then Theorem 5 becomes a generalization of the well known criterion for asymptotic stability of the zero equilibrium state of time-invariant linear systems [5, p. 76].

ACKNOWLEDGEMENT. This research was financially supported by the Science Fund of Serbia, grant number 0401E, through the Institute of Mathematics, Belgrade, Serbia, under the contract $0401 \mathrm{E}$, while the author was at the Faculty of Mechanical Engineering, Belgrade, Serbia, Yugoslavia.

\section{APPENDIX A-I. DEFINITIONS OF STABILITY DOMAINS}

Definition $A-1$. A set $J$ of the states of the system (3.1ab) has the domain $D_{s}(J)$ of stability if and only if for every mepsilon $\in] 0,+\infty\left[\right.$ there is a neighborhood $D_{s}(\varepsilon ; J)$ of the set $J$ such that $\underline{x}\left(t ; x_{0}\right)$ belongs to the $\varepsilon$-neighborhood $B_{\varepsilon}(J)$ of the set $J$ for all $t \in\left[0,+\infty\left[\right.\right.$ provided only that $x_{0} \in D_{s}(\varepsilon ; J)$ and that $D_{s}(J)=U\left[D_{s}(\varepsilon ; J): \varepsilon \in\right] 0,+\infty[\quad]$

Definition $A-2$. A set $J$ of the states of the system (3.1ab) has the domain $D_{a}(J)$ of attraction if and only if there is a neighborhood $D_{a}(J)$ of the set $J$ such that $\lim \left\{\rho\left[\underline{x}\left(t ; x_{0}\right), J\right]: t \rightarrow+\infty\right\}=0$ provided only that $x_{0} \in D_{a}(J)$.

Definition A-3. A set $J$ of the states of the system (3.1ab) has the domain $D(J)$ of asymptotic stability if and only if it has both the domain $D_{s}(J)$ of stability and the domain $D_{a}(J)$ of attraction and $D(J)=D_{s}(J) \cap D_{a}(J)$.

\section{A-II. PROPERTIES OF STABILITY DOMAINS}

Lemma A-1. If the system (1) possessing the Weak Smoothness Property has the domain D(J) of asymptotic stability of a set $J$ then

$$
\text { - } D_{a}(J) \subseteq D_{s}(J) \text { and } D_{a}(J)=D(J) \text {. }
$$

Proof. Let the system (3.1ab) possess the Weak Smoothness Property and have the domain $D(J)$. Then it has also the domains $D_{s}(J)$ and $D_{a}(J)$ (Definition A-3). Let $x_{0} \in D_{a}(J)$. Then, Definition A-2 and (i-b) and (ii) of the Weak Smoothness Property imply $\max \left\{\rho\left[\underline{x}\left(t ; x_{0}\right), J\right]: t \varepsilon\left[0,+\infty[\}-m\left(x_{0}\right)<+\infty\right.\right.$. Hence, for $\varepsilon \in\left[m\left(x_{0}\right),+\infty\left[, \underline{x}\left(t ; x_{0}\right) \in B_{\varepsilon}(J), \forall t \varepsilon\left[0,+\infty\left[\right.\right.\right.\right.$, which proves $x_{0} \in D_{s}(\varepsilon ; J)$, hence, $x_{0} \in D_{s}(J)$ and $D_{a}(J) \subseteq D_{s}(J)$. The last result and $D(J)=D_{s}(J) \cap D_{a}(J)$ prove $D_{a}(J)=D(J)$.

\section{REFERENCES}

1. BHATIA, N. P. and SZEGÖ, G. P., Dynamical Systems: Stability Theory and Applications, Springer-Verlag, Berlin, 1967.

2. GRUJIC, Lj. T., "Sets and Singularly Perturbed Systems," Systems Science, Vol. 5, No. 4, 1979, 327-338.

3. GRUJIC, Lj. T., "Concepts of Stability Domains," in Serbo-Croatian, Automatika, Zagreb, Vol. 26., No. 1-2, 1985, 5-10.

4. LA SALLE, J. P., "The Stability of Dynamical Systems," SIAM, Philadelphia, 1976.

5. LYAPUNOV, A. M., "The General Problem of Stability of Motion," in Russian, Kharkov Mathematical Society, Kharkov, 1892; French translation: "Problème Général de la stabilité du mouvement," Ann. Fac. Toulouse, Vol. 9, 203-247; also in: Annals of Mathematics Study, No. 17, Princeton University Press, Princeton, 1949.

6. VANELLI, A. and VIDYASAGAR, M., "Maximal Lyapunov Functions and Domains of Attraction for Autonomous Nonlinear Systems," Automatica, Vol. 21, No. 1, 1985, 69-80.

7. ZUBOV, V. I., Methods of A. M. Lyapunov and Their Application, P. Nordhoff Ltd., Groningen, 1964. 


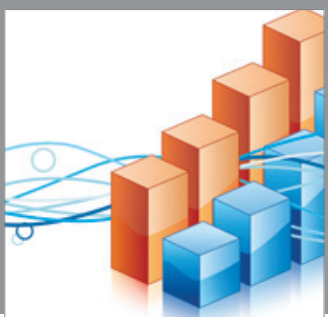

Advances in

Operations Research

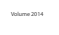

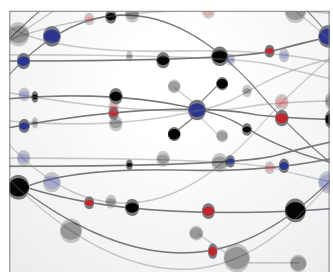

\section{The Scientific} World Journal
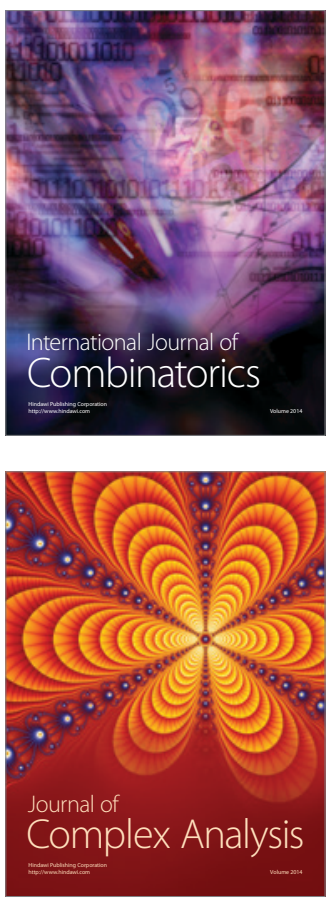

International Journal of

Mathematics and

Mathematical

Sciences
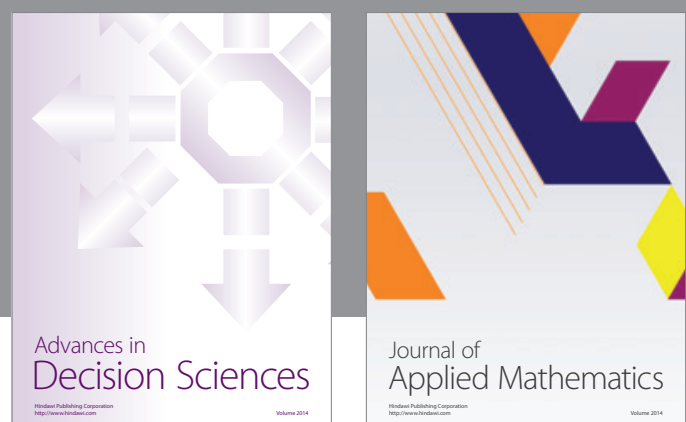

Journal of

Applied Mathematics
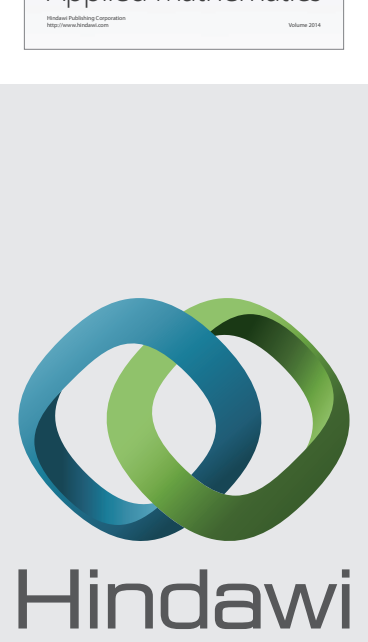

Submit your manuscripts at http://www.hindawi.com
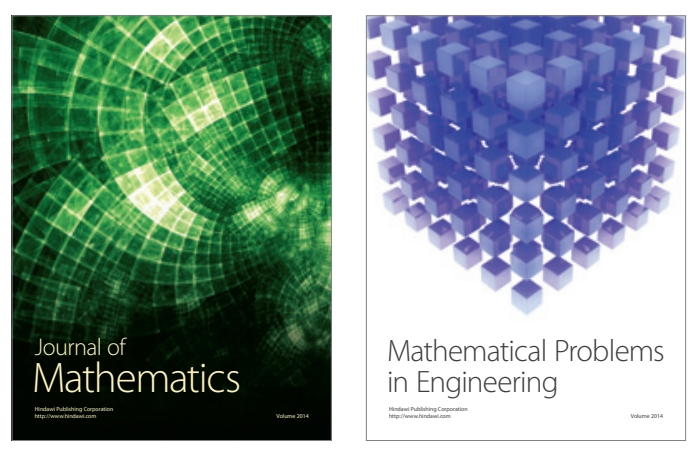

Mathematical Problems in Engineering
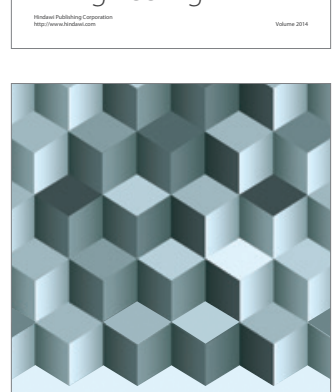

Journal of

Function Spaces
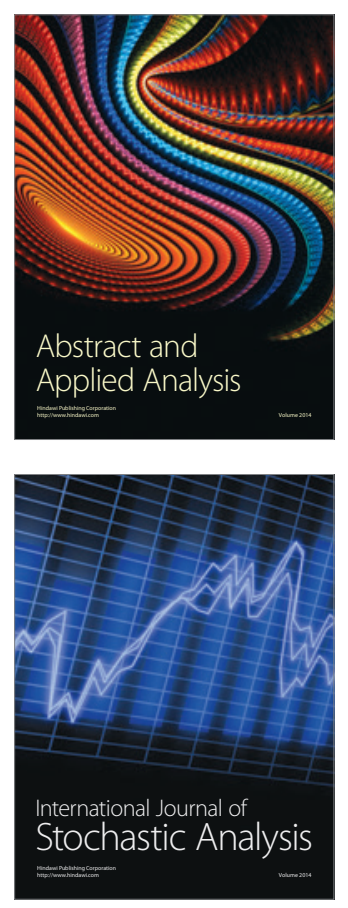

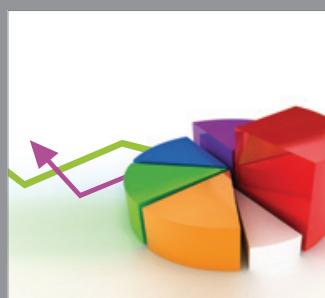

ournal of

Probability and Statistics

Promensencen
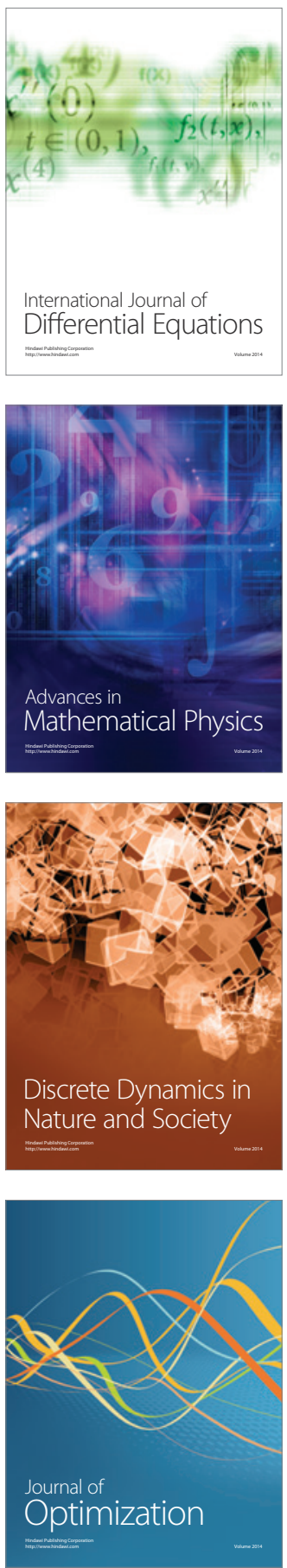